Methods Were studied restrospectively the medical records from these patients.

Results Out of the 46 cases, $60,8 \%$ were younger than 7 years, $41,30 \%$ were originated from urban environment; 27 cases were bilateral inguinal hernia; 14 presented various preoperative status/comorbidities (occlusive intestinal syndrome, acute dehydration, visceral adherence), 8 various surgical complications (bleeding, anaemia, scrotal swelling); the average length of stay was 3,69 days. Laparoscopic repair was significantly more frequent in patient younger than 7 years from urban environment (p 0,001), but also with significantly more frequent comorbidities $(p 0,04)$ and post surgical complications (p 0,003). Unilateral inguinal hernia develop fewer complications $(\mathrm{p} 0,03)$ and require a shorter length of stay $(p 0,008)$. The patients without comorbidities presented a significant shorter length of stay $(\mathrm{p}$ $0,05)$. The absence of surgical complications shortened significantly the lengh of stay ( $\mathrm{p} 0,01)$.

Conclusions Laparoscopic inguinal hernia repair may be considered a safe procedure, with fewer complications and shorten length of stay.

\section{PO-0911 HERLYN-WERNER-WUNDERLICH SYNDROME AND DISTAL VAGINAL ATRESIA. TWO PARTICULAR CASES}

${ }^{1} \mathrm{R}$ Cavaco Fernandes, 'E Blesa Sánchez, 'S Sanjuan Rodriguez, ${ }^{2} \mathrm{~N}$ Fuentes Bolaños. ${ }^{1}$ Paediatric Surgery, Hospital Materno Infantil, Badajoz, Spain; ${ }^{2}$ Paediatrics, Hospital Materno Infantil, Badajoz, Spain

\subsection{6/archdischild-2014-307384.1532}

Background and aims Müllerian anomalies are rare, causing malformations of the female reproductive system as the HerlynWerner-Wunderlich Syndrome (HWWS) and distal vaginal atresia. These patients are usually asymptomatic until menarche. There may be associated with renal and urinary tract anomalies. We describe two cases of young girls with occluded vagina associated with urethrovaginal fistula.

Cases presentation The first patient is a 3-years-old asymptomatic girl with prenatal diagnosis of left renal agenesis. Ultrasonography, performed 6 months prior to consultation, identified an anechoic cyst in hypogastrium. On genital examination, distal vagina occluded by a protruding membrane over the vaginal introitus. Magnetic resonance revealed a left paravesical formation suggestive of hydrocolpos. By the transmembrane puncture, urine was aspired. Laparoscopy confirms uterus didelphys. Membrane resection enable two hemivaginas. Based on the association of these anomalies, the diagnosis of HWWS was made. Girl present constant loss of urine and cystoureterography performed reveals urethrohemivaginal fistula. The second patient is a 4-years-old girl with occluded vagina suggesting distal atresia. Saline injected through the membrane eliminates by urethra. Cystoureterography confirms urethrovaginal fistula.

Conclusions The diagnoses of HWWS (uterus didelphys, unilateral low vaginal obstruction and ipsilateral renal agenesis) also known by OHVIRA and distal vaginal atresia in infancy and early childhood are unusual. The reported cases represent examples off premenarche diagnosis. In both patients, we detected urethrovaginal fistula, association not reported before in literature. Early diagnosis allows appropriate therapeutic management and prevents subsequent complications.

\section{P0-0912 SHORT BOWEL STATE: DOES AUTOLOGOUS GASTROINTESTINAL RECONSTRUCTION REDUCE CATHETER-RELATED BLOOD STREAM INFECTIONS?}

R Coletta, N Lansdale, BA Khalil, A Morabito. Paediatric Autologous Bowel Reconstruction and Rehabilitation Unit (Pabrru), Royal Manchester Children's Hospital, Manchester, Uk

\subsection{6/archdischild-2014-307384.1533}

Background and aims Catheter-related blood stream infections (CRBSI) occur frequently in Short Bowel Syndrome (SBS) children on parenteral nutrition (PN). Central venous catheter (CVC) complication and complete loss of central venous access are indication for intestinal transplantation. Autologous gastrointestinal reconstruction surgery (AGIR) is mandatory in any chronically $\mathrm{PN}$-dependent patient when there is substantial bowel dilation to reduce bacterial translocation. We reviewed patients who underwent lengthening surgery and calculated the rate of CRBSI pre and post surgery.

Methods PN dependent children with SBS were identified. Inclusion criteria were CVC for PN administration pre and postoperatively, CVC removed after weaned off $\mathrm{PN}$ and having gained enteral autonomy. CRBSI episodes were defined as temperature above $38.0^{\circ} \mathrm{C}$, along with positive blood culture microbiological infection from the CVC.

Results Nineteen patients were identified (male $\mathrm{n}=13$ ). Median gestation was 35 (33.5-36.5) completed weeks and birth weight $2080 \mathrm{~g}$ (1725-2374). Ten patients underwent tapering enteroplasty, eight Longitudinal Intestinal Lengthening and Tailoring (LILT) procedure, and one Serial Transverse Enteroplasty (STEP) procedure. Median duration of PN was 5.3 months (2.96.6) pre and 9.0 months (4.2-10.9) post surgery. A total of 115 septic episodes were confirmed (70 prior to surgery and 45 post surgery). The total rate of catheter related sepsis was significantly lower after AGIR compared to before it $(\mathrm{p}=0.016)$.

Conclusions CRBSI frequency in PN dependent patients with dilated bowel reduces after AGIR. AGIR appears associated with significantly reduced frequency of CRBSI in PN dependent children with bowel dilatation. These findings warrant further exploration in larger, preferably controlled studies.

\section{PO-0913 A REVIEW OF CUFFED VS. UNCUFFED ENDOTRACHEAL TUBES IN CHILDREN}

${ }^{1} \mathrm{D}$ Crankshaw, ${ }^{2} \mathrm{M}$ Entwistle. ${ }^{1}$ Medicine, Lancaster University, Lancaster, UK; ${ }^{2}$ Anaesthetics, Royal Lancaster Hospital, Lancaster, UK

\subsection{6/archdischild-2014-307384.1534}

Background and aims The use of cuffed endotracheal tubes in paediatric patients is still a controversial topic. This paper aims to investigate whether cuffed or uncuffed tubes should be used in children under the age of 8 based on the literature that is currently available on this topic. Currently there are no guidelines on this topic.

Methods literature review.

Results The results of the first four studies reviewed all show significant results in favour of use of a cuffed endotracheal tube and a change in current practice. None of the studies reviewed showed that patients were more likely to suffer injury as a result of using a cuffed tube. This is important as the review covers a variety of patients including those undergoing elective operations, burn patients and those who are critically ill. All of the 
studies reviewed demonstrated potential benefits of introducing cuffed tubes into practice as they reduced exchange rate, air leaks and the costs associated with the anaesthetic gases used; without a significant increase in the rates of post-extubation stridor or failed extubation.

Conclusion In conclusion cuffed tubes are shown to decrease the need for multiple intubations, reduce costs and are not shown to increase adverse effects in children of all ages.

\section{PO-0914 POSTOPERATIVE HYPERALGESIA IN CHILDREN AFTER ONCOLOGY SURGERY CORRELATES WITH RAISED LEVEL OF CORTISOL, INTERLEUKIN 6 (IL6), IL8 (IL8) AND C-REACTIVE PROTEIN (CRP)}

D Dmytriiev. Intensive Care, Vinnitsa National Medical University, Vinnitsa, Ukraine

\subsection{6/archdischild-2014-307384.1535}

Background The mechanisms contributing to postoperative hyperalgesia $(\mathrm{PH})$ in children are multifactorial. Recent evidence suggests a potential pathogenetic role for inflammation.

Objective To examine the relationship between serum concentrations of inflammatory mediators, cortisol (hydrocortisone) and PA after oncology surgicy.

Methods Prospective observational study involving children with $\mathrm{PH}$ after oncology surgery and normal controls. All patients after operation received adequate analgesic therapy (continuous infusion opioid analgesics). Blood samples were taken at birth from peripheral venous blood, at $8 \mathrm{~h}, 24$ and $42 \mathrm{~h}$ for cytokines, cortisol and CRP after surgical procedure. The area of hyperalgesia for punctuate mechanical stimuli around the incision was measured $48 \mathrm{~h}$ after the operation with a hand-held von Frey filament. For statistical analysis 2 tests were used.

Results 26 children (12,2 +/- 2,4 years) with PA and 20 controls $(12,8+/-3,1$ years) were enrolled. 14/26 (53,8\%) children with $\mathrm{PH}$ required mechanical ventilation. Children with $\mathrm{PA}$ had more than threefold higher serum levels of interleukin 8 (IL8) than the controls $(\mathrm{p}<0.05)$. At $8 \mathrm{~h}, 24 \mathrm{~h}$ and $42 \mathrm{~h}$, serum IL6 and CRP were 2.43- fold higher in neonates than the controls group $(\mathrm{p}<0.003)$. All patients with $\mathrm{PH}$ had significantly $(\mathrm{p}<0.001)$ higher plasma cortisol levels over control group (mean $+/-\mathrm{SD}$, $464.42+/-56.40$ vs. $202.21+/-37.30 \mathrm{micromol} / \mathrm{l}$ on $8 \mathrm{~h}$; $752.02+/-96.4$ vs. $308.12+/-100.1 \mathrm{micromol} / \mathrm{l}$ on $24 \mathrm{~h}$; $600.04+/-62.3$ vs. $302.8+/-51.2 \mathrm{micromol} / \mathrm{l}$ in $42 \mathrm{~h}$ ).

Conclusion This study demonstrated that postoperative hyperalgesia syndrome is associated with raised blood levels of proinflammatory mediators and cortisol, suggesting that inflammation contributes to the PA.

\section{PO-0915 THERAPEUTIC OPTIONS FOR CONDUCTIVE HEARING LOSS IN CHILDREN WITH CLEFT PALATE}

'S Franchella, 'MR Barillari, ${ }^{1} \mathrm{R}$ Bovo, ${ }^{1} \mathrm{~A}$ Martini, ${ }^{2} \mathrm{~A}$ Franchella. ${ }^{1}$ University Hospital, Otosurgery Unit, Padua, Italy; ${ }^{2}$ Maternity and Childhood, Pediatric Surgery, Ferrara, Italy

\subsection{6/archdischild-2014-307384.1536}

Background and aim Cleft lip and palate is a common congenital malformation correlated with otological disorders like Eustachian tube dysfunction, otitis media with effusion and conductive hearing loss correlated with speech disorders.

Methods In our study we evaluate the therapeutic options for the conductive hearing loss in these children, such as: middle ear ventilation tube insertion, hearing aids and adenoidectomy.
We conducted a retrospective chart review on 19 patients who underwent last follow-up during 2013, aged between 1 and 16 , affected by cleft palate. We identified 3 cases of TreacherCollins syndrome, 2 cases of CHARGE syndrome, 2 case sof Pierre Robin, 1 case of Goldhenar and 1 case of Di George syndrome. In 9 cases the cleft palate was isolated. In 14 cases we conducted phoniatric and logopaedic evaluations and 10 of these showed a speech disorder.

Results We found a conductive hearing loss in 12 of the 19 children. 2 of these patients used hearing aids with an improvementof speech performance; 1 patient underwent adenoidectomy for the appearance of sleep apnea but he modified his quality of voice with hypernasality and nasal emission after surgery; finally 9 children had spontaneous resolution of otitis media with effusion.

Conclusions In conclusion we found that the more effective way to resolve the problem of conductive hearing loss and the resulting speech disorder is to use the hearing aids until the resolution of the hearing loss, which normally occurs around 7 years old.

\section{PO-0916 MANAGEMENT OF GIANT CONGENITAL MELANOCYTIC NEVI: A SINGLE CENTRE 20 YEARS EXPERIENCE}

E Cesca, A Cazzuffi, A Franchella. Maternity and Childhood, Pediatric Surgery, Ferrara, Italy

\subsection{6/archdischild-2014-307384.1537}

Background and aim Giant congenital melanocyticnevus (GCMN) is a pigmented lesion present at birth with wide extent on the skin surface. This study aimed to assess the management of these lesions, which remains controversial and needs to take into consideration the perceived risk of melanoma, the patient's age, the cosmetic outcome, the surgical complexity, and the anesthesiological risk.

Methods This was a retrospective analysis and review of all records of children observed at the Paediatric Surgery Department oh the University-Hospital of Ferrara between 1991 and 2011 and treated for GCMN.

Results Twelve patients (median age 7 years, range $0.5-14$ ) with GCMN were reported during the study period. Neurocutaneous melanosis was documented only once. Four patients underwent staged excision with grafting, two dermabrasion, and six implantation of skin dilator-expanded flap transfer. 2/12 lesions were macroscopically completely excised. Compliance to therapy was close to $100 \%$. metastatic melanoma from an unknown primary site and death occurred in 1/12 (mean follow-up, 14 years).

Conclusions Decision making process to produce a final treatment choice for GCMN can be really complex. Regardless the outcome of therapy, a close regular post-operative follow-upto prevent or exclude possible complications proved to be useful in children with GCMN.

\section{PO-0917 REPORTING HAZARDS IN A HOSPITAL RATED 'HIGH RISK'}

${ }^{1} \mathrm{E}$ Jesudason, ${ }^{2} \mathrm{~K}$ Holt, ${ }^{3} \mathrm{P}$ Hammond, ${ }^{4} \mathrm{~S}$ Ahmed. ${ }^{1}$ Child Health, University of Liverpool, Liverpool, UK; ${ }^{2}$ Founder, Patients First, London, UK; ${ }^{3}$ Patron, Patients First, Bristol, UK; ${ }^{4}$ Paediatric Surgery, Alder Hey Children's Hospital, Liverpool, UK

\subsection{6/archdischild-2014-307384.1538}

Background and aims Why are professionals unheard when healthcare failures harm children? We answer with the first report of whistleblowing by UK paediatric surgeons. 